\title{
Cinco claves para la implementación de programas de formación virtual: una propuesta desde la experiencia de tutoría
}

\author{
Five Keys to the Implementation of Virtual Training Programs: A Proposal \\ from the Tutoring Experience
}

Claudia Godoy-Orellana ${ }^{1}$

\begin{abstract}
RESUMEN
La formación virtual es una modalidad educativa que cada vez adquiere más valor, por la serie de ventajas que ofrece, tanto en términos de inversión financiera como de uso del tiempo, accesibilidad y flexibilidad para los/as estudiantes, logrando una cobertura que trasciende distancias. Es innegable que este tipo de formación ha logrado posicionarse como una alternativa de alto valor en una sociedad que busca aprender y especializarse sin sacrificar la vida profesional y personal. Desde la experiencia profesional como tutora virtual, he logrado identificar ciertos aspectos relevantes para una implementación exitosa de este tipo de experiencias formativas, las cuales he sistematizado y profundizado con el propósito de elaborar una propuesta de cinco claves para el desarrollo de programas de educación virtual. Estas claves abordan desde lo más básico, que es contar con un soporte tecnológico que responda al desafío, hasta lo más complejo, que es orientar el desarrollo de capacidades de los/as estudiantes, para lo cual es fundamental tener un pleno conocimiento del perfil de entrada de los/as mismos/as. Otras claves se relacionan con las capacidades de los/as docentes que implementan las actividades curriculares, la posibilidad de contar con tutores/as de apoyo y el rol de las coordinaciones académicas.
\end{abstract}

Palabras claves: educación virtual; formación virtual; ambiente virtual de aprendizaje; educación online; educación a distancia.

\begin{abstract}
Virtual training is an educational modality that increasingly acquires more value, due to the series of advantages it offers, both in terms of financial investment and use of time, accessibility and flexibility for students, achieving coverage than transcends distances. It is undeniable that this type of training has managed to position itself as a high-value alternative in a society that seeks to learn and specialize without sacrificing professional and personal life. From the professional experience as a virtual tutor, I have been able to identify certain relevant aspects for a successful implementation of this type of training experiences, which I have systematized and deepened with the purpose of

\footnotetext{
${ }^{1}$ Tutora virtual del Magíster en Educación, Universidad Cardenal Silva Henríquez, Santiago, Chile; magíster en Dirección y Liderazgo para la Gestión Escolar; claudia.godoy.o@gmail.com.
} 
REVISTA SABERES EDUCATIVOS, Nº 5, JULIO-DICEMBRE 2020

elaborating a proposal of five keys for the development of virtual education programs. These keys address from the most basic, which is to have technological support that responds to the challenge, to the most complex, which is to guide the development of student capacities, for which it is essential to have full knowledge of their entry profile. Other keys are related to capacities of teachers who implement the curricular activities, the possibility of having support from tutors and the role of academic coordination.

Keywords: Virtual education; virtual training; virtual learning environment; online education; long distance education.

\section{Educación virtual en el debate nacional: desafío y oportunidad}

Desde hace ya más de una década, el despliegue de ambientes virtuales de aprendizaje en la educación superior ha ganado espacio en multitud de planteles educativos, especialmente en la oferta de posgrados, como una propuesta que considera las características, demandas y limitaciones los/as estudiantes, lo que, además, ha cambiado el escenario laboral de los/as docentes de educación superior. En 2018, la revista Educación Virtual ya informaba sobre el aumento del e-teaching, brindando antecedentes sobre un crecimiento proyectado del $13 \%$ de las ofertas de empleo para docentes de educación superior, incluyendo las ofertas para la formación virtual.

Un ambiente virtual de aprendizaje parece, a simple vista, una solución que rompe barreras de distancia y tiempo, ofreciendo una alternativa cómoda para quienes buscan compatibilizar la actividad académica de posgrado con la vida laboral y personal. Sin embargo, lograr la fórmula que asegure la entrega de una formación de calidad, en contraste con la presencialidad y el contacto directo con el/la académico/a formador/a, es sin duda un desafío que -hoy más que nunca- puede marcar la diferencia entre ofertas formativas con repercusiones significativas en el desarrollo académico y profesional de los/as estudiantes.

Ya en 2003, la Unesco aportaba importantes reflexiones y propuestas para mejorar la educación a distancia en Chile, principalmente mediante la "introducción y uso de NTIC, y su articulación con los medios educativos ya existentes” (Instituto Internacional, 2003, p. 41). Se planteaba que el desafío era “dejar de pensar en el aprovechamiento de las tecnologías 
CINCO CLAVES PARA LA IMPLEMENTACIÓN DE PROGRAMAS DE FORMACIÓN VIRTUAL /

GODOY-ORELLANA

para la educación y empezar a trabajar en la Educación Virtual” (Instituto Internacional, 2003, p.42), invirtiendo en investigación y desarrollo, generando competencias y un cambio real de paradigma en torno al proceso educativo.

Hoy, y justamente en el marco de la pandemia de COVID-19 en Chile, el debate en torno a las posibilidades y limitaciones de la educación virtual ha cobrado especial relevancia, tanto a nivel de políticas públicas como en la actividad académica. Los medios de comunicación ofrecen también espacios de análisis que invitan tanto al sistema educativo, en todos sus niveles, como a los/as usuarios/as, a preguntarse si es hora de considerar esta forma de educación como una de las principales alternativas educativas y qué tan preparados/as estamos como país para hacernos cargo del desafío.

El diario digital El Mostrador entrevistó, en el marco de esta emergencia sanitaria, a académicos de diferentes planteles de educación superior, quienes expresan su preocupación frente a qué tan preparados estamos, como país, para entregar una formación virtual en diferentes niveles de enseñanza (Fajardo, 2020). En el artículo publicado, el académico de la Universidad Cardenal Raúl Silva Henríquez, José Albuccó, señala que “en la educación universitaria este es un recurso muy usado, aunque existen competencias que necesitan modelamiento con prácticas y capacitaciones efectivas y pertinentes para cada disciplina en las universidades". En el mismo artículo, académicos y académicas de la Pontificia Universidad Católica de Chile y de la Universidad de Concepción, aportan su reflexión en relación sobre cuán importante es que comencemos a tomar en serio la formación virtual, fortaleciéndola y preparando a docentes y estudiantes, con un cambio radical en el uso de las tecnologías.

En este marco, es de suma relevancia contar con la experiencia de quienes han estado involucrados/as en este tipo de procesos, recogiendo empíricamente aquellos aspectos que podrían darnos luces acerca de cómo lograr que los procesos de educación a distancia, o educación virtual, respondan a las necesidades de la sociedad actual, asegurando la entrega de un servicio de calidad.

Desde mi experiencia profesional como tutora virtual y coordinadora de formación a distancia en programas de posgrado, así como en la gestión pública de políticas orientadas al 
fortalecimiento de capacidades de liderazgo para la mejora escolar, he logrado sistematizar ciertos aspectos y condiciones que son esenciales para potenciar los ambientes virtuales de aprendizaje como escenarios académicos de alto valor, asegurando la calidad de los procesos de enseñanza e implementando los mecanismos necesarios para monitorear el avance de los/as estudiantes, en función de los aprendizajes que se busca desarrollar en la especialización o la maestría. Esta sistematización se traduce en cinco claves que favorecen la implementación efectiva de programas de formación virtual.

Para contextualizar dichos elementos, haré una breve revisión de las ventajas más reconocidas de la educación virtual, para luego profundizar en los riesgos que esta modalidad educativa conlleva.

\section{Ventajas y desventajas de la educación virtual}

Sin duda, una de las principales ventajas de la educación virtual es la gran flexibilidad que ofrece a el/la estudiante en relación con el uso del tiempo, el punto de acceso al contenido y la disponibilidad de información durante las 24 horas del día, con la posibilidad de repetir lecciones y acceder a amplios repertorios de recursos de aprendizaje. Incluso, considerando lo más doméstico, se evita el uso del tiempo personal para desplazamientos. García Aretio (2017), a partir del análisis de numerosa literatura que revisa las principales ventajas de la educación virtual, menciona una serie de beneficios que la sitúan como una alterativa sumamente valorable. Según este autor, nos encontraremos con que este tipo de educación permite, en primer lugar, que las instituciones puedan realizar una amplia oferta de cursos, superando barreras geográficas, democratizando las oportunidades educativas al abrirse a segmentos más vulnerables, resultando, así, más inclusiva. El estudiante puede estudiar en la intimidad, pero igualmente accediendo a oportunidades de "interactividad e interacción”, así como de "socialización, porque permite el trabajo en grupo y el cultivo de actitudes sociales; se permite el aprender con otros, de otros y para otros, a través del intercambio de ideas y tareas, y ello puede ser con personas muy distintas y distantes, lo que favorece la multiculturalidad" (p.13). 
CINCO CLAVES PARA LA IMPLEMENTACIÓN DE PROGRAMAS DE FORMACIÓN VIRTUAL /

GODOY-ORELLANA

El mismo autor, tras años de recopilación de estudios, incluyendo metaanálisis de diversas investigaciones realizadas, menciona otros factores positivos que hacen de este tipo de programas una alternativa efectiva: los repositorios o bibliotecas son de una dimensión "cósmica", se innova en los procesos de enseñanza y aprendizaje, y hay una inmediatez, multidireccionalidad y ubicuidad que favorecen los aprendizajes significativamente, entre muchos otros atributos.

Empero, existen desventajas bastante comunes y que se relacionan, por una parte, con el riesgo de caer en la pasividad por parte de los/as estudiantes, depositándose en estos la responsabilidad absoluta de mantener constancia en el estudio y, por otra parte, con los problemas de estabilidad de las plataformas que sostienen el ambiente virtual de aprendizaje y la conexión a internet.

Gonzalo Vitale (2020), profesional de la Subdirección de Desarrollo Curricular del Instituto Profesional y Centro de Formación Técnica Duoc UC, señala que otra de las desventajas que existe a nivel nacional se relaciona con la brecha digital entre docentes o tutores/as y estudiantes, que son considerados/as nativos digitales (nacidos cuando ya existía la tecnología digital desarrollada).

Estos últimos superan en algunos casos a los docentes en conocimientos tecnológicos, lo que puede ser beneficioso en el caso de existir transferencia de conocimientos del uno al otro. Pero puede ser perjudicial también, dando posibilidades a estudiantes para copiar en pruebas usando WhatsApp u otro instrumento de transferencia de contenidos (Vitale, 2020).

En este escenario de contrastes, propongo a continuación cinco claves para la implementación de experiencias de educación virtual de calidad, las que creo son sumamente relevantes de considerar, ya sea en formación semipresencial o en aquella 100\% online. Estas claves abordan desde lo más básico, que es proveer la infraestructura adecuada, hasta lo más complejo de lograr, que es todo lo relacionado con los diferentes roles implicados en este tipo de experiencia formativa. 
REVISTA SABERES EDUCATIVOS, Nº 5, JULIO-DICEMBRE 2020

\section{Cinco claves para la implementación de experiencias de educación virtual de calidad}

\section{Lo básico: el soporte tecnológico y el ambiente virtual de aprendizaje}

Los ambientes virtuales de aprendizaje operan en servidores capaces de proveer una serie de funcionalidades y componentes que permitan a docentes y estudiantes trabajar en torno a los contenidos planificados, de forma fluida, atractiva y dinámica.

Es indispensable que las instituciones responsables de la formación cuenten con soportes tecnológicos robustos, tanto desde el punto de vista técnico como humano. Proveer una infraestructura técnica que permita instalar y utilizar una plataforma virtual de aprendizaje con el propósito académico definido, es lo básico. Contar con los/as especialistas profesionales y técnicos/as que garantizarán la continuidad del servicio, orientando a los/as usuarios/as en su adecuado uso y solucionando las dificultades que se presenten mientras, es sin duda uno de los factores esenciales a considerar, dado que los problemas asociados a este factor pueden ser causa de una insatisfacción por parte de los/as usuarios/as, derivando incluso en una pérdida de matrícula y otros dilemas administrativos y legales.

Un artículo publicado a través del medio digital de educación y aprendizaje en español Ined 21, por la autora Marta Merino (s.f.), menciona que "el objetivo principal de los sistemas de soporte es dar respuesta a las necesidades de los estudiantes y de esta manera aumentar el grado de satisfacción de los mismos". Ahora bien, esto es sumamente crítico también en el caso de los/as docentes, quienes requieren de un soporte efectivo que no dificulte su actividad pedagógica. La cantidad y tipo de consultas relacionadas con soporte técnico son variables y responder ágilmente a ellas debiera ser parte de uno de los indicadores de calidad del programa formativo. Por otra parte, la capacidad que docentes o tutores/as tengan para hacer uso de estos dispositivos y soportes también será fundamental: el ambiente virtual de aprendizaje es solo una herramienta y, como tal, carece de utilidad si no se sabe usar o si no se saca el máximo provecho a sus funcionalidades.

Para garantizar que un ambiente virtual de aprendizaje se transforme en un sistema altamente funcional a los propósitos definidos por las instituciones formadoras, una estrategia 
CINCO CLAVES PARA LA IMPLEMENTACIÓN DE PROGRAMAS DE FORMACIÓN VIRTUAL /

GODOY-ORELLANA

que ha demostrado, en mi experiencia, ser altamente eficaz es la de combinar instancias de formación sincrónicas y asincrónicas. Las clases online son bien valoradas por los/as estudiantes, quienes logran mayor compromiso y cercanía con el proceso de enseñanza, a través de la interacción directa con un/a docente. Estas se enriquecen cuando los/as docentes son capaces de mostrar presentaciones y otros recursos multimedia durante las clases, y también cuando son capaces de integrar variadas herramientas en la plataforma para realizar actividades diversas: chats, foros, encuestas, evaluaciones interactivas, entre otras.

Finalmente, se debe considerar que un ambiente virtual de aprendizaje óptimo basa su funcionamiento "en el principio de aprendizaje colaborativo" (Ecured, 2014,). Aquí es donde se tornan especialmente importantes las herramientas que permiten realizar aportes y resolver dudas, recibiendo retroalimentación de docentes y compañeros/as. Así, evolucionamos del clásico "texto en línea a un entorno interactivo de construcción de conocimiento" (Ecured, 2014).

\section{Docentes como protagonistas de la implementación pedagógica digital}

Intuitivamente y obviando las competencias propiamente disciplinares, podríamos asegurar que las capacidades básicas que un/a docente o tutor/a en línea debiera desarrollar se relacionan, por una parte, con el dominio de las tecnologías necesarias para transmitir conocimiento a través de un ambiente virtual de aprendizaje, desde la habilidad de manejar un computador hasta herramientas sincrónicas y asincrónicas para el aprendizaje. Por otra parte, son necesarias las habilidades instruccionales: desarrollar contenidos en la presencialidad no es lo mismo que en la virtualidad. Atraer, pero sobre todo mantener la atención de los/as estudiantes, mediante actividades que potencien tanto el autoaprendizaje como la colaboración entre pares, es un desafío no menor que requiere de experticia y constancia para evitar la pasividad por parte de los/as aprendices. La sistematicidad y rigurosidad en la carga de contenidos y actividades de aprendizaje, la incorporación de recursos multimedia y el diseño de evaluaciones sincrónicas y asincrónicas son ejemplos de lo que tanto la presencialidad como la virtualidad permiten. Sin embargo, en el ambiente digital, se debe desarrollar un lenguaje y una didáctica que logre mantener la atención de el/la 
estudiante mientras está inmerso en él, fomentando la dedicación hacia la actividad académica.

Compensar la falta de presencialidad y el contacto humano es algo que el ambiente virtual de aprendizaje no es capaz de lograr por sí mismo, por más contenidos y actividades bien diseñadas que se implementen. Los/as docentes tienen un rol protagónico: median entre los estudiantes y el ambiente virtual de aprendizaje, facilitando el proceso de aprendizaje desde el despliegue de sus habilidades curriculares y tecnológicas, y desde la dimensión humana, dado que humanizar la experiencia de aprendizaje también es un horizonte al cual los/as docentes debieran aspirar. Paulo Freire, uno de los principales referentes en educación humanista, confiaba plenamente

en el diálogo formador entre educador y educandos, hasta el punto de convertirlo en una estrategia pedagógica para concientizar al hombre situado, sujeto que mediante el diálogo se asume como un actor que se transforma a sí mismo, transforma las relaciones con el otro y, entre ambos, transforman el mundo para concretar un modo de vida más democrático y autónomo. (Pinto, 2004, p.1)

Esta idea debiera ser uno de los principios que guíe la interacción docente-estudiante y cobra más sentido aún en una modalidad de educación no presencial, donde un riesgo es, precisamente, deshumanizar la experiencia al ser mediada por la tecnología.

\section{Un plus: el apoyo profesional a los procesos de enseñanza y aprendizaje}

Una de las experiencias mejor valoradas por docentes y estudiantes es la posibilidad de contar con profesionales de apoyo en los procesos de enseñanza y aprendizaje virtual. Por lo general, los/as académicos/as que realizan clases presenciales incursionan en el mundo de la formación virtual adaptando sus métodos clásicos de enseñanza para dictar clases online o disponer de recursos que guíen el aprendizaje digital y autónomo por parte del/la estudiante. En ciertos programas, como lo son aquellos semipresenciales, el trabajo más fuerte se desarrolla en la presencialidad, depositando en el componente virtual todo aquello que se 
CINCO CLAVES PARA LA IMPLEMENTACIÓN DE PROGRAMAS DE FORMACIÓN VIRTUAL /

GODOY-ORELLANA

considera como tarea autónoma y que, más bien, refuerza los contenidos tratados en las sesiones presenciales. No obstante, cuando el componente virtual acapara un porcentaje de dedicación sustancialmente mayor al componente presencial, o cuando el programa es $100 \%$ a distancia, puede requerirse de apoyos profesionales para montar la infraestructura digital pedagógica, en función de asegurar interacciones que promuevan efectivamente el logro de aprendizajes de calidad. En pocas palabras, uno de los desafíos más relevantes de los docentes será generar, en el ambiente digital, interacciones con sus estudiantes y entre estos, tan productivas como la presencialidad, generalmente, lo permite.

Los/as profesores/as que preparan sus sesiones online, así como actividades de aprendizaje y evaluación, pueden potenciar el efecto educativo al contar con un/a tutor/a virtual de apoyo que mantenga contacto más estrecho con los/as estudiantes, orientándolos durante el proceso de educación a distancia y colaborando en el diseño pedagógico del curso.

En este sentido, creo acertado hablar de mi experiencia como tutora virtual del Magíster en Educación de la Universidad Cardenal Raúl Silva Henríquez, que en su diseño combina presencialidad con virtualidad. Los/as docentes realizan clases presenciales, de forma tradicional, pero en paralelo los estudiantes cuentan con el apoyo de tutoras/es online que complementan y enriquecen los procesos pedagógicos desde el ambiente virtual de aprendizaje, maximizando las oportunidades de aprendizaje de los/as estudiantes y actuando como canales de comunicación entre estos/as y los/as docentes, en relación con diversos temas que van surgiendo en el marco del proceso académico. Esto, a mi juicio, ha favorecido también la humanización del proceso de enseñanza y aprendizaje, facilitando espacios de interacción, retroalimentación y apoyo constantes.

En el marco de la emergencia sanitaria del año 2020, cuando el confinamiento obligó a suspender actividades lectivas presenciales en esta universidad, el componente virtual se volvió el protagonista de las formas de enseñar y aprender: la alianza de docentes con tutoras/es online ha resultado en un factor virtuoso de éxito, en cuanto los/as docentes, que podrían no estar tan familiarizados con estas nuevas formas de enseñar, contaron con un apoyo exclusivo para desarrollar y complementar sus clases sincrónicas, por parte de profesionales altamente capacitados para desarrollar procesos de formación virtual. Docente 
REVISTA SABERES EDUCATIVOS, № 5, JULIO-DICEMBRE 2020

y tutor/a colaboran sistemáticamente para producir una experiencia en la cual los/as estudiantes se sientan cómodos/as, atendidos/as, desafiados/as y acompañados/as en su proceso de aprendizaje, en un escenario de incertidumbre y cambio respecto a las formas de trabajar y vivir.

Contar con asistentes profesionales en un rol de tutor/a se transforma así en un plus que maximiza la posibilidad de éxito del programa formativo, contribuyendo a potenciar los canales de comunicación y socialización, promoviendo la actividad de los/as estudiantes desde el apoyo motivacional y pedagógico, y fortaleciendo el diseño e implementación pedagógica.

\section{Una coordinación académica capacitada para afrontar el desafío de la virtualidad}

Los y las profesionales que coordinan la actividad académica de facultades y direcciones de educación de pregrado y postgrado, deben atender a los desafíos de la educación virtual en igual medida que atienden los procesos de formación presenciales. Las propuestas educativas a distancia deben ser igualmente eficaces en relación con el logro de egresados/as altamente capacitados/as, lo que desafía a las coordinaciones a atender una multiplicidad de formatos para la enseñanza, en función de las necesidades de los/as estudiantes, procurando mantener altos niveles de satisfacción con la experiencia académica y apoyando la labor docente desde una gestión que asegure la existencia de todas las condiciones necesarias para que los procesos de enseñanza y acompañamiento fluyan sin inconvenientes.

En el marco de los estudios sobre la formación a distancia o virtualizada,

se cuestiona si realmente los profesores tienen una adecuada preparación para enfrentar este reto en el actual contexto en que transcurre el proceso de formación inicial y continua. Esto genera una contradicción entre los principios didácticos de la educación a distancia con el uso de las TIC y el currículo concebido para su formación. (Juca, 2016) 
CINCO CLAVES PARA LA IMPLEMENTACIÓN DE PROGRAMAS DE FORMACIÓN VIRTUAL /

GODOY-ORELLANA

Los y las profesionales a cargo de la coordinación de estos programas debieran ocuparse especialmente de este desafío: contar con docentes altamente capacitados/as en este tipo de procesos -ya sea mediante la capacitación o el reclutamiento-, romper barreras culturales y educativas en relación con esta modalidad de enseñanza y conducir procesos académicos de calidad asegurando un soporte tecnológico efectivo, serán tareas ineludibles de las cuales hacerse cargo. Esto implica desarrollar un arduo trabajo en torno a la definición de estándares de calidad claros a los cuales hacer seguimiento, desde una estructura organizativa robusta, una gestión que innova permanentemente y que se atreve a ser disruptiva en una sociedad que está en permanente evolución, consumiendo y exigiendo contenidos y experiencias cada vez más tecnologizados. Esto exige a los equipos coordinadores una gran capacidad de adaptación, flexibilidad y aprendizaje: las formas de enseñar y aprender evolucionan, y la tecnología también. Ser jinete de los avances en materia de TIC requiere de una dedicación y actualización permanentes.

\section{Conocer el perfil de el/la estudiante a nivel de entrada, orientando el desarrollo de capacidades hacia el perfil de egreso}

La quinta y última clave para asegurar una implementación efectiva de experiencia de educación a distancia, según mi apreciación tras años de trabajar en esta materia, se relaciona con las características de los/as estudiantes y cuán bien conocemos su perfil de entrada. Esto nos permitirá definir las brechas a acortar y acercarnos al perfil de egreso definido, de forma más eficiente.

En general se selecciona a los/as estudiantes que formarán parte de la matrícula de los programas de formación virtual, en base a antecedentes como el currículum vitae y cartas de presentación, acompañados -en ocasiones- de recomendaciones por parte de jefaturas o académicos/as que puedan brindar información sobre las capacidades y desempeño de quienes postulan a un programa de especialización o magíster. En este marco, podemos hipotetizar mucho sobre las capacidades o el perfil ideal de un/a estudiante de posgrado, pero es escaso el control que la institución formadora tiene sobre esa variable al momento de su ingreso. 
En este escenario, es clave que los/as postulantes conozcan a priori aquellas características que se espera manifiesten durante el proceso formativo, permitiéndoles reflexionar acerca de cómo desarrollar o fortalecer las capacidades necesarias para responder a las exigencias de un programa virtual de formación. Asimismo, conocer dichas características, por parte de las instituciones formadoras, será clave para potenciarlas, sobre todo a través de las primeras experiencias de aprendizaje que se implementen. El rol de los/as tutores/as virtuales de apoyo a la docencia puede ser altamente relevante en este aspecto, como facilitadores/as de aprendizajes globales en virtud del avance académico.

Respecto del perfil de estudiantes que debemos buscar o potenciar, será esencial contar con personas altamente motivadas con el proceso académico y que sean capaces de autorregular su aprendizaje, demostrando disposición a participar en la experiencia formativa con responsabilidad, disciplina, autonomía y capacidad para colaborar con otras personas en una interacción respetuosa, nutritiva y profesionalizante. Se requiere de una atención constante, en cuanto los ambientes virtuales de aprendizaje están en constante intercambio de información, con plazos acotados para la realización de actividades y evaluaciones. Pese a que una de las virtudes de las plataformas virtuales es contar con acceso a la información “24/7”, esto no significa que los/as estudiantes pueden carecer de hábitos de planificación. De hecho, uno de los problemas más comunes que manifiestan los/as estudiantes es "no contar con tiempo suficiente" para responder con la carga académica. Las instituciones pueden apoyar a los/as estudiantes con orientaciones y herramientas que complementen la formación especializada, contribuyendo a la creación de hábitos adecuados en el uso del tiempo y el espacio, y brindando experiencias que estimulen el desarrollo de autonomía y colaboración.

\section{Conclusiones}

En conclusión, favorecer una implementación efectiva de una experiencia de educación virtual es una tarea sumamente desafiante, debido a la gran cantidad de variables a considerar tanto en el diseño como en la implementación de los programas formativos. 
CINCO CLAVES PARA LA IMPLEMENTACIÓN DE PROGRAMAS DE FORMACIÓN VIRTUAL /

GODOY-ORELLANA

Considerar estas cinco claves resulta esencial para lograr que los/as estudiantes adquieran una serie de capacidades de alto valor académico y profesional, que respondan a los desafíos de la sociedad actual. Que el soporte tecnológico sea robusto y estable es, sin duda, una condición de base para montar toda la infraestructura pedagógica de las ofertas virtuales de formación: sin soporte no hay virtualidad. No obstante, quienes dotan de sentido a estos programas son los/as docentes con habilidades curriculares, pero también tecnológicas, que logran hacer del proceso una experiencia humanizante. El apoyo por parte de tutores/as virtuales y coordinaciones académicas, tanto capacitadas como comprometidas con la experiencia virtual, son otros aspectos que es recomendable que fortalezcan las instituciones que ofertan educación a distancia. Por último, recordar que conocer los perfiles de entrada de los/as estudiantes será esencial para guiar su proceso de aprendizaje, entregándoles herramientas no solo disciplinares o propias de la especialización, sino también capacidades personales e interpersonales que los acerquen al éxito académico y profesional.

Para cumplir con estas cinco claves, las instituciones de educación superior que ofertan programas de formación virtual debieran ocuparse especialmente, desde los equipos que lideran y coordinan estas iniciativas, no solo de contar con profesionales que sean expertos/as en las especialidades impartidas, sino también de desarrollar y/o fortalecer sus capacidades tecnológicas para un aprovechamiento óptimo de las herramientas digitales que hoy los/as estudiantes, en gran medida, dominan como nativos/as digitales. El mundo avanza -y es una de las lecciones que nos deja la emergencia sanitaria en Chile- hacia una revalorización de este tipo de ofertas, encontrando en esta situación una oportunidad para romper paradigmas y propender hacia la búsqueda de una excelencia que trasciende las modalidades en que la enseñanza es impartida.

Otro desafío concreto es el de no perder el horizonte humanizante de la educación, en un proceso de actualización que obliga a tecnologizar cada vez más los procesos pedagógicos, dotando de apoyos tanto a docentes como estudiantes que interactúan en ambientes virtuales de aprendizaje. Serán las instituciones formadoras quienes podrán contribuir significativamente, tanto desde la investigación como desde la praxis, al enriquecimiento de las experiencias de formación virtual, con miras hacia una sociedad que busca más y mejores oportunidades de crecimiento académico y profesional. 


\section{Referencias}

EcuRed, Ambiente Virtual de Aprendizaje (2014). Disponible en: http://www.ecured.cu/index.php/Ambiente_Virtual_de_Aprendizaje

Fajardo, M. (3 de marzo de 2020). Expertos analizaron debilidades de la educación a distancia y coinciden en que "no estamos preparados". El Mostrador. Recuperado de https://www.elmostrador.cl/cultura/2020/03/30/educacion-a-distancia-paramillones-en-plena-crisis-sanitaria-expertos-advierten-que-no-estamos-preparados/

García Aretio, L. (2017). Educación a distancia y virtual: calidad, disrupción, aprendizajes adaptativo y móvil. RIED. Revista Iberoamericana de Educación a Distancia, 20(2), 9-25. doi: http://dx.doi.org/10.5944/ried.20.2.18737

Instituto Internacional de la UNESCO para la Educación Superior (2003). La Educación virtual en Chile: historia, estado del arte y proyecciones; hacia el conocimiento de la realidad nacional en materia de uso de tecnología aplicada a la educación superior. Unesco. Biblioteca Digital. Recuperado el 30 de abril de: https://unesdoc.unesco.org/ark:/48223/pf0000140393

Juca, F. (2016). La educación a distancia, una necesidad para la formación de los profesionales. Revista Universidad y Sociedad, 8(1), 106-111. Recuperado de http://scielo.sld.cu/scielo.php?script=sci arttext\&pid=S2218$\underline{36202016000100016 \& \operatorname{lng}=\text { es\&tlng=es. }}$

Merino, M. (s.f.). Los sistemas de soporte en entornos virtuales de aprendizaje. Ined 21. Recuperado de: https://ined21.com/los-sistemas-de-soporte-en-entornos-virtualesde-aprendizaje/

Nuevas tendencias en la educación virtual. Revista Educación Virtual. Recuperado de: https://revistaeducacionvirtual.com/seccion/pedagogia/amp 
CINCO CLAVES PARA LA IMPLEMENTACIÓN DE PROGRAMAS DE FORMACIÓN VIRTUAL /

GODOY-ORELLANA

Pinto, R. (2004). Paulo Freire: un educador humanista cristiano en Chile. Pensamiento Educativo. Revista de Investigación Educacional Latinoamericana, 34(1), 234-258.

Vitale, G. (2020). Los desafíos de la educación a distancia. Duoc UC. Observatorio Duoc. Recuperado de:

http://www.duoc.cl/observatorio/los_desafios_de_la_educacion_a_distancia 